\title{
Analysis of the Correlation between Activity of the Suprahyoid Muscles, Infrahyoid Muscles and the New VFSS Scale in Stroke Patients with Dysphagia
}

\author{
Hyun-Jin Lee, OT, PhD • Kyoung-Don Kim, PT, $\mathrm{PhD}^{1 \dagger}$ \\ Department of Occupational Therapy, Kyungwoon University \\ ${ }^{1}$ Department of Physical Therapy, Dongju College
}

Received: July 4, 2018 / Revised: July 9, 2018 / Accepted: August 22, 2018

(c) 2018 J Korean Soc Phys Med

\begin{abstract}
| Abstract |
PURPOSE: This study was conducted to investigate the correlation between the activity of suprahyoid muscles, infrahyoid muscles and swallowing ability in stroke patients with dysphagia.
\end{abstract}

METHODS: The subjects of this study were 120 patients who were diagnosed with CT or MRI stroke and had swallowing disorder at Daegu Metropolitan General Hospital from August 2014 to February 2017. The suprahyoid and infrahyoid muscle activity was measured in patients with dysphagia and the new videofluoroscopy swallowing studies scale (new VFSS scale) was used for evaluation of swallowing function. Correlation analysis was conducted using the measured data.

RESULTS: The activities of suprahyoid muscles were 325.8 (\%RVC) on average, while the average infrahyoid muscle activity was 302.65 (\%RVC) and the average value of

$\dagger$ Corresponding Author : Kyoung-Don Kim doniee1595@gmail.com, http://orcid.org/0000-0001-7173-2004 This is an Open Access article distributed under the terms of the Creative Commons Attribution Non-Commercial License (http://creativecommons.org/licenses/by-nc/3.0) which permits unrestricted non-commercial use, distribution, and reproduction in any medium, provided the original work is properly cited. the new VFSS scale was 31.52. The total for oral organs was 3.62 on average and that for pharynx organs was 28.30 on average. The activity of the suprahyoid muscles showed a significant positive correlation with the activity of the infrahyoid muscles, but a significant negative correlation with the total oral phase, total pharyngeal phase and total new VFSS scale $(p<.01)$. The activity of the infrahyoid muscles showed a significant negative correlation with the total oral phase, total pharyngeal phase, and total new VFSS ( $p<.01)$. CONCLUSION: Based on the results of this study, it is necessary for researchers to consider the infrahyoid and suprahyoid muscles when conducting swallowing rehabilitation.

Key Words: Dysphagia, Stroke, Swallowing

\section{Introduction}

Swallowing is defined as a complex muscular movement in which food is taken orally and delivered to the digestive system, such as to the stomach through the pharynx and esophagus (Logemann, 1998). The failure of food intake to reach the stomach through the mouth and esophagus is known as dysphagia (Kawashima et al., 2004).

While dysphagia occurs because of complex damage around muscles related to the corticobulbar tract and 
swallowing, it has been reported that recovery of swallowing after stroke is affected by factors such as recovery of muscular strength of the swallowing muscles (Robbins et al., 2005). Accordingly, for functional training of dysphagia, increases in muscular power and amount are necessary in addition to changes in neural plasticity (Won, 2012).

The suprahyoid muscles reduce food residue; therefore, there is a risk of aspiration caused by moving the hyoid bone and larynx in an anterior direction and a forward direction and by helping the upper esophageal sphincter open after swallowing (Shaker et al., 2002). A number of previous studies have suggested that once the suprahyoid muscles are enhanced through neck bending exercise or by neuromuscular electrical stimulation, swallowing ability is strengthened (Shaker et al., 1997; Easterling et al., 2000; Freed et al., 2001; Mun, 2013).

The PNF neck bending exercise is said to enhance infrahyoid muscles as well as suprahyoid muscles (Adler et al., 2000). Based on this theory, Kim (2015) subjected dysphagia patients to PNF neck bending exercise and the shaker exercise and compared the activities of the suprahyoid muscles, infrahyoid muscles and improvements in swallowing ability to before both exercises were performed. The results confirmed that both exercises improved the activity and swallowing ability of the suprahyoid muscles and the infrahyoid muscles of the patients.

Previous studies have suggested that strengthening of the suprahyoid muscles improves swallowing ability (Shaker et al., 1997; Easterling et al., 2000; Freed et al., 2001; Mun, 2013), but they failed to reveal the correlation between the suprahyoid muscles and swallowing. In addition, Kim (2015) did not reveal any relationship between the infrahyoid muscles that help improve swallowing, the suprahyoid muscles or swallowing ability. Therefore, this study was conducted to investigate the exact correlation between the activity of the suprahyoid muscles, the infrahyoid muscles and swallowing ability in stroke patients with dysphagia.

\section{Methods}

\section{Subjects and Term}

The research subjects of this study were patients diagnosed with stroke through $\mathrm{CT}$ or MRI at 00 General Hospital in Daegu Metropolitan City between August 2014 and February 2017. A total of 120 subjects who understood the purposes and backgrounds of this study were selected. The standards of selection of participants of this study were as follows. First, those who were diagnosed with stroke through CT or MRI were selected. Second, those who were diagnosed with dysphagia after development of stroke were selected. Third, those who won MMSE-K marks at 24 or above were selected. Fourth, people without internal diseases other than stroke were selected (Table 1).

The study was screened by the Clinical Test Screening Committee hosted by Daegu Fatima Hospital. Letters of consent from patients and their guardians were acquired before the study. The IRB (institutional review board) screening number is DFH14ORIO020.

\section{Tools of Measurement}

\section{1) Measurement of muscle activity upon neck bending}

To measure the activities of suprahyoid and infrahyoid muscles, a surface electromyogram consisting of four channels (Biopac Systems, Inc., Goleta, CA, USA) was used. Data recorded during swallowing by a research subject were subject to filtering and other signal processing using Biopac Student Lab Pro (Biopac System Inc. CA. Santa Barbara. USA. The electrodes of the suprahyoid muscles were attached in the direction of muscle fiber extension at a distance of $1 \mathrm{~cm}$ from the midline on the chin of the participant onto the area of the neck that is $1 \mathrm{~cm}$ parallel, outside of the midline of the thyroid cartilage 
Table 1. General Characteristics of the Experimental Group

\begin{tabular}{lc}
\hline \multicolumn{1}{c}{ Variables (units) } & Experimental group $(\mathrm{n}=120)$ \\
\hline Sex (male/female, n (\%)) & $61(50.8 \%) / 59(49.2 \%)$ \\
Age (year) & $60.65 \pm 8.30$ \\
Height (cm) & $160.76 \pm 8.20$ \\
Weight (kg) & $64.52 \pm 10.53$ \\
Duration (month) & $7.70 \pm 4.10$ \\
MMSE-K & $27.52 \pm 22.11$ \\
Type of stroke (infarction/hemorrhage, n (\%)) & $75(62.5 \%) / 45(38.5 \%)$ \\
Paretic side (right/left, $\mathrm{n} \mathrm{( \% ))}$ & $69(57.5 \%) / 51(42.5 \%)$ \\
\hline
\end{tabular}

$\mathrm{M} \pm \mathrm{SD}$ : Mean \pm Standard Deviation

(Valenzuela et al., 2006). The areas to which electrodes were attached was cleared of hair and then cleaned with medicinal alcohol to reduce measurement errors. Next, a three-poled (positive-ground-negative) EMG surface electrode $\mathrm{Ag} / \mathrm{AgCl}$ (Biopac, diameter $2 \mathrm{~cm}$ ) was attached to the venter at which muscles are most. The sampling rate of the electromyogram signals was $1024 \mathrm{~Hz}$. To remove noise from the measured electromyogram signals, raw materials were subjected to band pass filtering around 10 $\mathrm{Hz}-200 \mathrm{~Hz}$, followed by rectification, after which they were smoothed at RMS 20 ms (Lee, 2014). The experiment was conducted while the participant was sitting on a chair with a backrest and swallowing $2 \mathrm{ml}$ of water. With \%RVC (reference voluntary contraction) used in this study, the mean EMG values obtained while the participant was resting were set as the reference muscle contraction values. In this study, the muscle activity of the suprahyoid muscles was set as the reference muscle contraction value and the muscle activities were observed through the mean value obtained from the three swallowing activities completed by the participant (Mun, 2013).

\section{2) New videofluoroscopy swallowing studies (new VFSS scale)}

To test the swallowing functions, new VFSS scales were measured. The new VFSS scale, which is based on videofluoroscopy swallowing studies (VFSS), consists of 14 factors ranging from 0 to 100 . Higher marks are related to more severe dysphagia. The researchers determined the new VFSS scale values based on VFSS read by an expert in rehabilitation medicine. Seven items pertained to oral organs, palatal obturator, formation of food lumps, mastication, swallowing apraxia, tongue-palate contacts, level of earlier loss of food lumps and length of time required to pass through oral organs. Additionally, seven items related to the pharynx, the remains of epiglottic vallecula, reduction of laryngeal elevation and epiglottis closing, remains of pyriform sinus, larynx membrane formation after swallowing, pharynx delay time length, pharynx passage time length and absorption (Hong et al., 2012). This new VFSS scale measure was made in consideration of relative odds that occur to oral organs and pharynx organs observed upon video inspection of swallowing. The sensitivity of this measure was $72.0^{-}$ $81.0 \%$, while the singularity was $70.0-81.0 \%$, showing a significant correlation with severity of absorption (Jung et al., 2005).

\section{3) Analytical Method}

The statistical program SPSS for Windows 18.0 was used to analyze the results, which were described based on the means and standard deviation. The general 
Table 2. Descriptive Statistics of Experimental Group

\begin{tabular}{lc}
\hline \multicolumn{1}{c}{ Variables (units) } & $\begin{array}{c}\text { Experimental } \\
\text { group ( } \mathrm{n}=120)\end{array}$ \\
\hline \% RVC & $325.85 \pm 140.21$ \\
Suprahyoid & $302.65 \pm 143.43$ \\
Infrahyoid & \\
New VFSS (oral phase) (point) & $.10 \pm .43$ \\
Lip closure & $.65 \pm 1.24$ \\
Bolus formation & $.53 \pm 1.36$ \\
Mastication & $.05 \pm .27$ \\
Apraxia & $1.19 \pm 2.12$ \\
Tongue to plate contact & $.82 \pm 1.00$ \\
Premature bolus loss & $.45 \pm 1.07$ \\
Oral transit time & \\
New VFSS (pharyngeal phase) (point) & $2.76 \pm 1.83$ \\
Residue in the valleculae & $31.52 \pm 16.49$ \\
Reduced laryngeal elevation and epiglottic closure & $6.60 \pm 3.99$ \\
Residue in the pyriform sinuses & $5.13 \pm 3.92$ \\
Coating of pharyngeal wall after swallow & $6.60 \pm 3.99$ \\
Pharyngeal delay time & $.70 \pm 1.27$ \\
Pharyngeal transit time & $.50 \pm 1.66$ \\
Aspiration & $6.00 \pm 4.85$ \\
\hline & $3.62 \pm 5.11$ \\
Total oral phase (point) & $28.30 \pm 14.52$ \\
& \\
\hline
\end{tabular}

$\mathrm{M} \pm \mathrm{SD}$ : Mean \pm Standard Deviation

New VFSS : new video-fluoroscopic swallowing study scale

characteristics of the research subjects, new VFSS scale and activities of suprahyoid muscles and infrahyoid muscle were subjected to descriptive statistics, while Pearson's correlation analysis was applied to compare correlations among activities of the suprahyoid muscle, infrahyoid muscle and the new VFSS. Upon correlation analysis, since the difference between the values of the data was large, analysis was performed after the data were standardized. A $p<.05$ was considered to indicate significance.

\section{Results}

1. Descriptive statistics of muscle activity and swallowing

Based on the test results, the average activity of the suprahyoid muscles was 325.8 (\%RVC), while that of the infrahyoid muscles was 302.65 (\%RVC). Additionally, the average VFSS scale value was 31.52 . The total average for oral organs was 3.62, while that of pharynx organs was 28.30 (Table 2).

\section{Correlations among activities of suprahyoid} muscles and infrahyoid muscles and swallowing

The activity of the suprahyoid muscles showed a significant positive correlation with the activity of the infrahyoid muscles, but a significant negative correlation with total oral phase, total pharyngeal phase and total new VFSS scale ( $<.01)$ (Table 3). The activity of the infrahyoid muscles showed a significant negative correlation with the total oral phase, total pharyngeal phase, and total new VFSS scale $(\mathrm{p}<.01)($ Table 3$)$.

\section{Discussion}

This study was conducted to investigate the correlation between the activity of the suprahyoid muscles, infrahyoid muscles and swallowing function in stroke patients with dysphagia.

\section{Research method}

The new VFSS scale has been used in many previous studies to measure the function of swallowing (Eun et al., 2010; Hong et al., 2012; Kim, 2015). The data used for correlation analysis among the new VFSS scale results were total oral phase, total pharyngeal phase sum, and total sum.

The activity of the suprahyoid muscles, the infrahyoid muscles and the New VFSS scale was measured during the evaluation stage before the participants were rehabilitated. 
Table 3. Correlation Between Measurements

\begin{tabular}{|c|c|c|c|c|c|}
\hline Variables (units) & $\begin{array}{c}\text { Zscore } \\
\text { (Suprahyoid } \\
(\% \text { RVC }))\end{array}$ & $\begin{array}{c}\text { Zscore } \\
\text { (Infrahyoid } \\
(\% \text { RVC) })\end{array}$ & $\begin{array}{c}\text { Zscore } \\
\text { (Total Oral } \\
\text { phase (point)) }\end{array}$ & $\begin{array}{c}\text { Zscore } \\
\text { (Total Pharyngeal } \\
\text { phase (point)) }\end{array}$ & $\begin{array}{c}\text { Zscore } \\
\text { (Total New } \\
\text { VFSS (point)) }\end{array}$ \\
\hline $\begin{array}{c}\text { Zscore } \\
\text { (Suprahyoid }(\% \mathrm{RVC}))\end{array}$ & 1 & $.648^{* *}$ & $-.245 * *$ & $-.753 * *$ & $-.718 * *$ \\
\hline $\begin{array}{c}\text { Zscore } \\
(\text { Infrahyoid }(\% \mathrm{RVC}))\end{array}$ & & 1 & $-.253 * *$ & $-.673 * *$ & $-.649 * *$ \\
\hline $\begin{array}{c}\text { Zscore } \\
\text { (Total Oral phase (point)) }\end{array}$ & & & 1 & $.320 * *$ & $.567 * *$ \\
\hline $\begin{array}{c}\text { Zscore } \\
\text { (Total Pharyngeal phase (point)) }\end{array}$ & & & & 1 & $.959 * *$ \\
\hline $\begin{array}{c}\text { Zscore } \\
\text { (Total New VFSS (point)) }\end{array}$ & & & & & 1 \\
\hline
\end{tabular}

Since there was a large difference between the muscle activity value and the new VFSS scale value, correlation analysis was conducted after data standardization.

This study investigated 120 participants for 2 years and 6 months, and there were no dropouts because there was no intervention period.

\section{Results}

The suprahyoid muscles showed a significant negative correlation with total oral phase, total pharyngeal phase, and total new VFSS, indicating that, as the activity of the suprahyoid muscles increases, the scores of these factors decreases. In other words, increased activity of the suprahyoid muscles and the infrahyoid muscles improves swallowing function. Previous studies have not revealed a correlation between the suprahyoid muscles and the new VFSS scale; therefore, direct comparison is impossible. However, the results of this study are considered to theoretically support those of previous studies that showed increased activity of the suprahyoid muscles caused the forward and upward movement of the hyoid bone to increase (Easterling et al., 2000; Kim and Lee, 2009; Mun, 2013).

In this study, the infrahyoid muscles showed a significant negative correlation with the total oral phase, total pharyngeal phase, and total new VFSS. This could theoretically support the results of a study conducted by Kim (2015) that suggest increasing the activity of the infrahyoid muscles and suprahyoid muscles improves swallowing function. However, previous studies of swallowing rehabilitation have focused on the suprahyoid muscles. The infrahyoid muscles are located below the hyoid bone and involved in movement of the hyoid bone. The present study revealed a significant positive correlation between the infrahyoid muscles and the suprahyoid muscles. Therefore, the results of the present study indicate that researchers need to study the infrahyoid muscles as well as the suprahyoid muscles as they relate to swallowing rehabilitation.

It should be noted that this study was limited in that muscle activity was only assessed when participants were drinking water and not when they were eating. Therefore, future studies should investigate the activation of suprahyoid muscles and infrahyoid muscles when participants actually eat. It is also necessary to conduct additional studies of patients with severe dysphagia who have cognitive problems. 


\section{Conclusion}

To investigate the correlation between muscle activity of the suprahyoid muscles, the infrahyoid muscles and swallowing function, this study measured suprahyoid muscles and infrahyoid muscles and the new VFSS scale values of 120 patients with dysphasia. The activity of the suprahyoid muscles showed a significant positive correlation with the infrahyoid muscles, but a significant negative correlation with the total oral phase, total pharyngeal phase, and total new VFSS. Additionally, the activity of the infrahyoid muscles showed a significant negative correlation with total oral phase, total pharyngeal phase, and total new VFSS. These findings indicate it is necessary for researchers to consider both the infrahyoid muscles and the suprahyoid muscles when developing methods of swallowing rehabilitation.

\section{Acknowledgements}

This paper was written with a research fund provided by Kyungwoon University.

\section{References}

Adler SS, Beckers D, Buck M, et al. PNF in Practice. Springer. 2000.

Easterling C, Kern M, Nitschke T, et al. Restoration of oral feeding in 17 tube fed patients by the Shaker Exercise. Dysphagia. 2000;15(2):105-9.

Eun SJ, Kim SG, Hong JR. The Usefulness of Video Fluoroscopic Swallowing Study in Post-Stroke Dysphagia Patients. Journal of the Korean Society of Radiology. 2010;4(3):19-25.

Freed ML, Freed L, Chatburn RL, et al. Electrical stimulation for swallowing disorders caused by stroke. Respiratory care. 2001;46(5):466-74.

Hong DK, Kyoung KS, Yu DH. The effect of Shaker exercise on swallowing function of stroke patients. Journal of Korean Society of Occupational Therapy. 2012; 20(3):55-66.

Jung SH, Lee KJ, Hong JB, et al. Validation of clinical dysphagia scale: based on videofluoroscopic swallowing study. Journal of Korean Academy of Rehabilitation Medicine. 2005;29(4):343-50.

Kawashima K, Motohashi Y, Fujishima I. Prevalence of dysphagia among community-dwelling elderly individuals as estimated using a questionnaire for dysphagia screening. Dysphagia. 2004;19(4):266-71.

Kim DK, Lee SJ. The Effects of Shaker Exercise and Electrical Stimulation on Swallowing Ability ofthe Stroke Patients with Dysphagia caused by Failure of Upper Esophageal Sphincter Relaxation : Case Report. the Journal of Korean Academy of Dysphagia Rehabilitation. 2009;1(1):55-60.

Kim KD. The effect of PNF neck-flexion exercise on swallowing function of chronic stroke patients. Doctor's Degree. Daegu university. 2015.

Lee MH. The effect of cervical stabilization exercise on respiration function of stroke patients. Doctor's Degree. Daegu university. 2014.

Logemann JA. The evaluation and treatment of swallowing disorders. Current Opinion in Otolaryngology \& Head and Neck Surgery. 1998;6(6):395-400.

Mun TH. The effects of shaker exercise and neuromuscular electrical stimulation therapy on the swallowing function: on the post-stroke patients with dysphasia. Master's Degree. inje university. 2013.

Robbins J, Gangnon RE, Theis SM, et al. The effects of lingual exercise on swallowing in older adults. Journal of the American Geriatrics Society. 2005;53(9):1483-9.

Shaker R, Easterling C, Kern M, et al. Rehabilitation of swallowing by exercise in tube-fed patients with pharyngeal dysphagia secondary to abnormal UES opening. Gastroenterology. 2002;122(5):1314-21.

Shaker R, Kern M, Bardan E, et al. Augmentation of deglutitive 
upper esophageal sphincter opening in the elderly by exercise. American Journal of Physiology. 1997; 272(6):1518-22.

Valenzuela S, Baeza M, Miralles R, et al. Laterotrusive occulusal schemes and their effect on supra- and infrahyoid electromyographic activity. The Angel Orthodontist.
2006;76(4):585-90.

Won YS. The effects of oropharyngeal exercise combined with tongue pressure training protocol on swallowing function in stroke patients with dysphagia. Journal of Special Education \& Rehabilitation Science. 2012;51(2):57-71 